\title{
Fuzzy sets make fuzzy logic
}

\section{Artificial intelligence has become all the rage, often by government sponsorship.The concept of fuzziness, while interesting, points to few distinctive benefits. Understanding remains the need.}

Ir is a common and legitimate complaint about computers that they are maddeningly literal. One who keeps a list of references to the literature on a computer and who enters one author under the name of JONES cannot afterwards hope to retrieve it by calling JOSNE. And naturally, if JONES is mistakenly entered as JOSNE, and the perpetrator of this simple misspelling does not realize what has happened, the entry may never be retrieved. This puts computers in a poor light compared with people, who can usually tell at a glance when one entry in a printed document is a misprint for some other.

What can be done to remedy these infuriating deficiencies? Formally, at least, there is very little difficulty. It would now be an undergraduate exercise in some computer science course to follow each vain search for an alphabetical data base by the construction of all anagrams (meaningful or not) of the object word and to institute a search for them. The only cost will be measured in computer-time, no great obstacle these days. The drawback is that such a solution, or even the refinement in which (in principle) some empirical knowledge of which mistypings are most common is used to construct a hierarchy of anagrams, is exceedingly inelegant. And the solution of the problem of finding a misspelling of an unknown index-word would require the construction of all anagrams of all possible index-words or otherwise a wooden search through every cell of the computer memory.

No wonder that the search is on for more effective solutions of this kind of problem. The banner under which the search is undertaken is by now familiar as "artificial intelligence", Al for short. Components of it are to be found in publicly sponsored development programmes such as the British Government's response to the report of the Alvey committee on information technology, the collaborative programme of industrial research in Japan known (wrongly) as the Fifth-Generation Computer Programme, the European Esprit programme and elsewhere. In passing, in Britain, it is curious that AI should so quietly have become respectable a decade after a report by Sir James Lighthill assured the Science Research Council (as it then was) that it had better not touch the topic with a barge-pole.

The objective is to embody in machines some of the attributes of human perception and subsequent analysis. So much is un- controversial. One thing that might be agreed in advance is that solutions of the problem of accurate perception in which the deficiencies of existing machinery are made good by extra hidden programming, the construction of all the anagrams of JONES for example, will not count. The justification for that disdain is that they are bound to be particular solutions of particular problems.

A small step forward would be to replace the search for JONES by a search for some four-letter word derived from the original by the omission of one character. Many would argue that this is in reality a simulation of what people do when scanning a list of index-entries - much of the information on the printed page is redundant, rarely is every letter essential. The snag is that such a procedure might well recover the name JOHNS as well as JONES, but that would rarely be a serious difficulty.

Such a way of tackling the problem of AI seems to have inspired the now fashionable concern with what is called fuzziness, the attempt to build imprecision into programs and even into the machines which such programs instruct. Plainly there are many practical benefits in such developments. Computerized control systems whose input is blessed with spurious accuracy, for example, are capable of hunting (in the technical sense) for an ideal that does not exist; built-in imprecision, perhaps by means of running time averages, is well known to be advantageous in such circumstances. What is much less clear is whether the great flowering of the concept of fuzziness is likely to be as rewarding.

At least one recent book (Advances in Fuzzy Sets, Possibility Theory and Applications, Paul P. Wang (ed.), Plenum, New York, \$55) suggests that the movement may have gone too far, at least in its claims to have important practical applications. The notion of a fuzzy set, the invention of L.A. Zadeh of the University of California in 1965, is an entirely uncontroversial extension of respectable mathematical concepts. The conventional definition of a set of points on the interval of some line presupposes that the points themselves are well defined, if not numerically but then by some rule. It is perfectly proper, and interesting, to ask how the properties of sets must be modified if imprecision is built in; plainly quantities representing measure (distance) which may be conventionally additive may lose that convenient property when the sets are fuz- zy (but that may be just the property that AI requires).

What remains unclear is whether the concept of fuzziness as thus defined is capable of being applied in computer problems in ways that are inherently different from much more homespun concepts, for example that the positions of all points or the values of all numbers may be uncertain but nevertheless related to a "true" value by some probability distribution. The devotees of fuzzy theory who contribute to Wang's volume (one third of whom are from the People's Republic of China) insist that their new craft is distinct from probability theory, in practice as well as in principle. The crucial test will be whether they can write computer programs that are markedly more intelligent than can be devised by embedding all numbers in probability distributions, at this level the analogue of the search for JONES which takes account of all anagrams of the word.

While this issue is undecided, it is perhaps fortunate that the prospects for true AI are not wholly bound up in fuzzy sets, and the fuzzy logic by which they must be manipulated. Genuinely parallel computing networks, perhaps operating out of synchrony with each other, offer scope for powerful experiments. There are also opportunities for programmers following some of the interesting demonstrations of the past few years. By now, for example, it is clearly possibly to provide programs with such a detailed model of how the human body works, and with such a wealth of physiological data, that the machines which carry them can match the performance of human physicians at the diagnosis of diseases drawn from a restricted set. In several working systems of this kind, the machines do follow human physicians in forming hypotheses and checking them against the data they have been given, making judgements along the way about the significance of different elements of the data, sometimes conflicting. As yet, however, nobody seems to have claimed that machine physicians can better the performance of the human kind.

In the circumstances, it is forgivable that many people regard AI as a field of research, not of development. The task of understanding and then simulating the visual system shows what conceptual difficulties lie ahead. Not the least of these is semantic. When will a machine be able to say "I see JONES!'?

John Maddox 